\title{
EMERGENCY MEDICAL SYSTEMS ANALYSIS BY SIMULATION AND OPTIMIZATION
}

\author{
Pedro Marinho Sizenando Silva \\ Luiz Ricardo Pinto \\ Federal University of Minas Gerais \\ Presidente Antônio Carlos Av, 6627 \\ B. Horizonte, MG, 30161-010, BRAZIL
}

\begin{abstract}
Several works published in the medical and operational research literature demonstrate the existence of a direct relationship between the response time of rescue units and the probability of the survival of victims involved in accidents. This work presents the development of a methodology integrating the discreteevent simulation techniques and optimization procedures for medical emergency system analysis. The main components of the methodology are presented in detail and used for the evaluation of the emergency medical system of the city of Belo Horizonte in Brazil.
\end{abstract}

\section{INTRODUCTION}

\subsection{Problem Formulation}

In Brazil the emergency medical system is known as SAMU, a Portuguese acronym for Urgency Medical Assistance Service. The service was established in 2003 by the Federal Government and is available 24 hours a day. The service is executed by professionals of health teams, such as doctors, nurses and assistants. They attend several types of emergencies, including those of trauma, pediatric, surgical, gynecological, obstetric and mental health emergencies (Timerman et al. 2006). The basic function of the system is to assist in an organized and fast way (using a minimum of resources) for all emergency events that require medical services. The service begins with the phone call communicating the event and finishes when the patients are assisted in the ambulance and either released or hospitalized. The service should use the most appropriate resources, such as ambulances and medical teams, to assist and transport the patients and to direct them to the most appropriate hospital (Takeda et al. 2001).

An emergency medical system can be seen as a system with a unique queue and multiple servers which is controlled by one central location. Moreover, the probability distribution of time service is a function of the available ambulances from the moment the call arrives at the centre. In addition to this dependence, the time of service also depends on the location of each ambulance. These characteristics of the process makes the analytical modeling of this queuing system very difficult since the location of the servers and the space distribution of the incidents influence the time of travel, this being a significant component of the time of the service (Fitzsimmons 1971).

The Brazilian emergency medical system follows the French model, with previous assistance and whereby the victims are assisted in the place where the event occurred. In other words, the system is not only for the transport of the patients.

Many authors provide information about the existence of a direct relationship between the waiting time for the service and the probability of the victims' survival. Cummings (1989) affirms that for patients with cardiac arrest, every minute of delay in the rescue reduces the chance of survival to $7-10 \%$. Cum- 


\section{Silva and Pinto}

mins et al. (1985) also observed that if the procedure of cardiopulmonary resuscitation is made within 5 minutes from the instant of the cardiac arrest and the defibrillation process within 10 minutes, the survival percentage for admissions to the hospital is $30 \%$, but otherwise decreases to less than $7 \%$.

\subsection{Literature Review}

Brailsford (2007) has affirmed that simulation models for health studies can be classified into three groups. The first one constitutes models of the human body that represent biological processes; the second group is one of models whose focus is modeling patient flows in the system to analyze, for example, the bottlenecks of the system; the last one is that of strategic models where the objective is to analyze the strategic planning of the organization such as the configuration of the service system, analyzing the projections regarding the number of patients in the system, etc.

Nowadays, the changes in the health services around the world has compelled managers to use new tools in the planning and evaluation of processes in order to improve the quality level of the systems. In addition to the pressure to deliver services of better quality using restricted resources, the growing use of quantitative measures to monitor and manage the medical services has been implicated in the need for a better understanding of the results that occur when one service has changed before its implementation (Eldabi and Young 2007).

The first efforts to apply the concepts of simulation in healthcare date back to the 60's with the work of Fetter and Thompson (1966) to develop models to solve problems relating to the scheduling of appointments for patients. The authors were able to identify important variables in evaluating the use of the medical team, such as the rate of the arrival of patients, early or late arrivals, delays, scheduling intervals and stops due to the shift change of doctors.

The problem in analyzing the emergency services can be approached in different ways, e.g. the queuing theory (Singer and Donoso 2008), the location of bases (Toregas et al. 1971), the design of specific areas of coverage (Chuck and ReVelle 1974), simulation (Shih and Su 2003) and the hypercube model (Oliveira Gonçalves 2004), among others.

An early application of simulation techniques for modeling and the analysis of emergency medical services was developed in the 70's by Fitzsimmons (1971). A simulation model was implemented using the SIMSCRIPT language. The model assisted the managers of health systems in the evaluation of the existing emergency medical services or services in the planning stages.

Shih and Su (2003) also developed a model of discrete event simulation to evaluate the emergency medical services in the city of Taipei, Taiwan. The study focused on 23 hospitals which qualified for emergency care and 36 service centers. The service, at the time of the study, worked in conjunction with the fire department providing basic medical care (in the case of less serious incidents) by sending a basic care unit. The model was able to identify and propose improvements which resulted in a $50 \%$ reduction in the delay time between the arrival of an incoming call and the dispatch of an ambulance, and decreased to less than $1 \%$ the probability of patients waiting to be rescued. Different policies for operating an emergency medical system can be evaluated through simulation techniques. Koch and Weigl (2003) presented a model based on discrete event simulation to study the logistics of transporting the rescue service of the Red Cross in Austria, comparing the policies of centralization and decentralization of the rescue units. The network traffic representative of the model was implemented in SIMAN due to the possibility of using graph algorithms integrated with the language to solve the shortest path problems. The results showed that there is potential for reducing the waiting time for patients and the distance travelled if the operational system is centralized. An interesting approach to emergency systems analysis is the combination of facility location models and simulation techniques for the analysis of the solutions generated. Aringhieri, Carello, and Morale (2007) developed an integer linear programming model for locating medical care units in Milan, Italy and a simulation framework for testing solutions. The ambulance redeployment problem was modeled by Maxwell, Henderson and Topaloglu (2009). In this case the problem was modeled as a Markov decision process in order to evaluate the performance of the allocation policies. A simulation 


\section{Silva and Pinto}

model and a dynamic programming work were also integrated to compute high-quality redeployment policies for Edmonton, Alberta.

\section{ASSUMPTIONS AND MODEL FORMULATION}

\subsection{Simulation Model}

For modeling, all the system processes for historical data were used $(1,734,416$ calls $)$, these being the basis for an estimation of the parameters of the model.

\subsubsection{Calls Arrival}

The calls come from all the city areas according to a Poisson process and are received in the control center, hereafter referred to as "Central", according to a FIFO rule. In order to model the spatial distribution of calls, the city was divided into regions and historical data was used to determine the behavior of the calls in each region. To determine the coordinates of the incidents, the city was mapped using the UTM (Universal Transverse Mercator) system coordinates. The UTM coordinates were also used to determine the exact location of the bases and service centers considered by the system and to make the calculation of travel time easier, based on the Euclidean distance between two points. The contour of each region was approximated using a polygon which matched with the limits of the region. Here, the coordinates of the incident were randomly distributed in each region and a procedure was developed to sample the spatial origin of each call.

\subsubsection{Central Processes}

In the real system, when the call makes evident the need to send an ambulance, a variety of information is collected and then the call is transferred to the doctors of the Central, who decide whether or not to send an ambulance. If the doctor decides that it is not necessary to send an ambulance, the call is finished. Otherwise, the call is moved to the dispatch sector, which chooses which ambulance will be sent to the place of the event. Therefore, the service in the Central is characterized by three main activities: i) the initial selection of calls by attendants to define its nature and eliminate joke calls, wrong calls and information calls; ii) medical analysis by the regulator doctor in order to decide if it is necessary to send an ambulance; iii) the dispatch process that chooses the ambulance and sends it to the place of event. The process begins when a call arrives in the call center and is received by one of the available attendants. After generating the call, the system determines its attributes using the historical data. These attributes concern: i) the origin of the call (UTM coordinates); ii) the medical nature of the call (general, trauma, gynecological, obstetric or psychiatric); iii) medical decisions (whether or not to send an ambulance).

\subsubsection{Ambulance Dispatch Policy}

The Brazilian emergency medical services work with two major types of ambulances: basic units (basic life support - BLS) and advanced units (advanced life support - ALS). The former are used in simpler events or to support an advanced unit, and the team consists of a rescuer driver and nursing assistant. Advanced units are only engaged in more complicated and serious cases, and their teams have a rescuer driver, a nurse and a doctor. The choice of what type of ambulance must be sent is made exclusively by the doctor on call at the Central. Once at the event site, the team sends new information to the Central and the doctor on call can send an advanced unit if necessary. In major Brazilian cities the emergency medical system operates in a decentralized way, composed of several bases located throughout the city with a certain number of ambulances in each one and a Central for receiving and dispatching calls. The choice of which ambulance will be dispatched is a fundamental process for reducing the response time of the system, this being influenced by several factors, including proximity and availability. Any representative 


\section{Silva and Pinto}

computing implementation of the emergency medical services must consider for all the time spent in various care processes in order to ensure that the unit allocated to a call is the one that actually will arrive first on the scene. In order to lead this implementation, a control array can be used to store in their cells all information relevant to the process of choosing the ambulance to be dispatched. Such information is stored for each ambulance and concerns the following:

- the time that the ambulance will be available;

- coordinates of the place where the ambulance will be available;

- the number of calls waiting for the ambulance;

- the type of ambulance.

To determine the travel time, a very simple approach widely used in the analysis of transport services and routing problems is the use of Euclidean distance, weighted by a correction factor, and taking into account specific features of the locality being studied, such as the traffic intensity and the configuration of the streets net (large avenues, intersections, one-way roads, etc.). The Euclidean distance between two points $A:\left(x_{A}, y_{A}\right)$ and $B:\left(x_{B}, y_{B}\right)$ is given by:

$$
D=\sqrt{\left(x_{A}-x_{B}\right)^{2}+\left(y_{A}-y_{B}\right)^{2}}
$$

This distance, in most real applications of transport, is a useful abstraction for the calculations and structure of the models. The reason for this is its simplicity for analytical representation and its unique characteristic. By using corrective coefficients, it is possible to make a mathematical relationship between the actual and Euclidian distances, thereby allowing a more realistic treatment of the applications (Novaes 1989).

According to Novaes (1989), in the case of an urban environment, there are several factors that affect the distance travelled, since the existence of one-way routes can increase the travel time. There are also restrictions on the crossroads of major avenues, requiring the vehicle to find a way to allow the crossing. The calculation of the travel time of the ambulance $T_{d}$ must take into account the Euclidean distance weighted by the correction factor and the average speed of the ambulance, according to relationship (2):

$$
\mathrm{T}_{\mathrm{d}}=\mathrm{D} * \mathrm{~F} / \mathrm{V}
$$

where $\mathrm{D}$ is the Euclidean distance, $\mathrm{F}$ is the correction coefficient and $\mathrm{V}$ is the average speed.

The calculation of the average speed of an ambulance is influenced by the intensity of traffic. This intensity is variable and time-dependent, resulting in an average value for each time range of the day. Once triggered, the system samples a priori the service time and the UTM coordinates where the ambulance will be available. After the service completion, the system determines, if any material replacement and ambulance maintenance is necessary based on historical data.

Hence, when a new call arrives, the release times for each ambulance will all be updated, even if an ambulance is in attendance. When the service is finished, the system checks to see if there is a waiting call, to decide its destination. If there is no call, the ambulance returns to the home base and updates the time and the coordinates of release. Initially, the dispatch procedure checks to see if there is a required type of ambulance. Then the choice of a specific ambulance is based on a lower response time. If the release time of the ambulance is greater than the current simulation time, this means the ambulance is in service. The time elapse between a call and the arrival of the ambulance is the response time, this being calculated by (3):

$$
\mathrm{T}=\mathrm{T}_{\mathrm{r}}-\mathrm{T}_{\mathrm{c}}+\mathrm{T}_{\mathrm{d}}
$$




\section{Silva and Pinto}

where $T$ is the response time, $T_{r}$ is the release time and $T_{c}$ is the current simulation time. $\left(T_{r}-T_{c}\right)$ is the waiting time until the ambulance is available and $T_{d}$ is the travel time between the place of release and the event site. If $T_{r}<T_{c}$ the ambulance is already available and the response time is $T_{d}$. The response time for each ambulance is stored in an ascending order and the first position informs which ambulance must be dispatched. This procedure is made for basic and advanced ambulances and the choice takes into account the type of ambulance required.

\subsubsection{Service at Local Site}

After dispatching the required ambulance, the team must be informed by radio or telephone about the location of the event and during the journey, complementary information about the event is sent to the team. In real systems, the best route can be chosen using a Global Positioning System (GPS) in order to eliminate the problems caused by an ambulance driver's lack of knowledge of a region. The arrival at the site does not always mean accessing the victim. Campbell et al. (1993) presented a study to determine the time between the arrival of the ambulance at the site and the medical team's moment of contact with the patient, additionally identifying the major factors that affect the displacement of the team during this time interval. When victims are trapped in wreckage or under building structures, it is necessary to have a rescue team that works efficiently and which is integrated with the healthcare team, in order to facilitate access to the victim; the service can be realised fully or partially in the local site and then the victim is moved to the ambulance. This time depends on the complexity of the situation, and involves factors such as the condition of the victim, the qualifications of the medical team, the team size, environmental conditions and service rules. In standard emergencies, there is usually only a single victim, but in trauma emergencies, especially in car crashes, this number may be higher. The same team can help two or more victims, before the arrival of another team but this situation may increase the time on site. The system should therefore evaluate this time as part of the day-to-day work. However, the time spent in the handling of the victim must be carefully analyzed for use as an efficiency factor.

\subsubsection{The Choice of the Destination Hospital}

In a well organized system, victims are removed to referral hospitals according to a predetermined schedule. Therefore, when Central reports that a victim is being transferred, the reception tends to work faster. In more severe cases, however, the victim may be referred to a service center, bearing in mind factors such as the proximity and nature of the event. In Brazilian cities, the emergency medical system works with Emergency Care Units for standard cases, with hospitals for serious medical trauma, obstetric and gynecological cases and with Mental Health Reference Centers for psychiatric cases. The problems arising from a lack of knowledge concerning the expertise of the physicians available in each hospital at every shift makes the choice of hospital more complex. Moreover, the choice of the center is always made by the doctor in the Central, not by the team that is performing the service.

\subsubsection{Materials Replacement}

The ambulance only works if all the materials are properly packaged and sanitized. The mean time between the materials' replacement in the emergency medical system of Belo Horizonte is 24 hours for basic ambulances and 12 hours for advanced units. In Belo Horizonte, the replacement is always carried out in the Central. The model estimates the moment of materials' replacement for each ambulance and simulates their stoppages to perform this task.

\subsubsection{Performance Parameters}

The response time is an appropriate performance measure to evaluate the accessibility of emergency medical services (Hong and Ghani 2006). Another parameter commonly used to analyze the performance of 


\section{Silva and Pinto}

the emergency services is the percentage of calls with service times below a predetermined time. According to Ball and Lin (1993), U.S. regulations for emergency medical services require that 95\% of the requests in an urban area must be served in less than 10 minutes, and in less than 30 minutes for rural areas.

\subsection{Optimization Model}

\subsubsection{Simulation Optimization}

Optimization for simulation focuses on the use of two techniques to improve the system analysis; the use of simulation to estimate the performance of a system usually requires a high computational time, and determining the optimal configuration is a very difficult problem to handle because of the need to evaluate the system performance for different configurations.

With the optimization for simulation problem, the objective function or constraints or both are responses that can only be evaluated by computer simulation, because these functions are only implicit functions of the decision parameters of the system and have a stochastic nature (Azadivar 1992).

\subsubsection{Model Formulation}

We considered the monthly costs of the operation of basic or advanced ambulances. The first step was to determine which bases should be used and how many units should exist in each base. Depending on the allowable ranges for each variable of the problem, the number of scenarios can be extremely high, making necessary the use of a heuristic solution. Here, the average response times must be calculated separately for each type of unit. This is done to prevent the optimizer from underestimating the number of advanced units (these being more expensive) to reduce the value of the objective function. Furthermore, this model allows the response criteria to be different for each type of ambulance, rendering the system more flexible. The optimization problem can be formulated as follows (Fu, 2002):

$$
\underset{\theta \in \Theta}{\operatorname{Min}} J(\theta)=E[C(\theta, \omega)]
$$

where $\theta$ represents the vector of input variables, which in this case would be the set of bases for the allocation and quantity of each type of ambulance in each base; $J(\theta)$ represents the objective function; $\omega$ represents the replication; $C(\theta, \omega)$ denotes the sample performance measure (in this case, the total cost); and $E[C(\theta, \omega)]$ is the expected value of $C(\theta, \omega)$. The constraints are:

$$
\begin{array}{ll}
R^{a}(\theta, \omega) \leq R T^{a} & \\
R^{b}(\theta, \omega) \leq R T^{b} & \\
N_{i}^{a}(\theta, \omega) \leq N A L S & \forall i \\
N_{i}^{b}(\theta, \omega) \leq N B L S & \forall i
\end{array}
$$

where $R^{a}(\theta, \omega)$ and $R^{b}(\theta, \omega)$ are the response times for the sample $\theta$ in replication $\omega$ for advanced and basic units respectively; $N_{i}^{a}(\theta, \omega)$ and $N_{i}^{b}(\theta, \omega)$ are the quantity of advanced and basic units allocated in each base $i$ at the sample $\theta$ and replication $\omega ; R T^{a}$ and $R T^{b}$ are the upper response times of the advanced and basic units; $N A L S$ and $N B L S$ are the upper bounds for the total units in each base (advanced and basic). To solve the problem, an optimizer and a simulator must work together; the optimizer uses metaheuristics to seek the candidate solutions while the simulator evaluates the performance of each one. 


\section{Silva and Pinto}

\section{THE CASE OF THE EMERGENCY MEDICAL SYSTEM OF BELO HORIZONTE}

Belo Horizonte has a total area of $330.954 \mathrm{~km}^{2}$ and a population of approximately 2,500,000 inhabitants. The city is divided into nine regions. The emergency medical system operates 24 hours a day and is operated by one Central and nineteen auxiliary bases throughout the city. The quantity of ambulances in each base are fixed. Altogether there are 23 ambulances (17 basic units (BLS), 5 advanced units (ALS) and one unit for psychiatric care). The spatial distribution of these units can be seen in Figure 1.

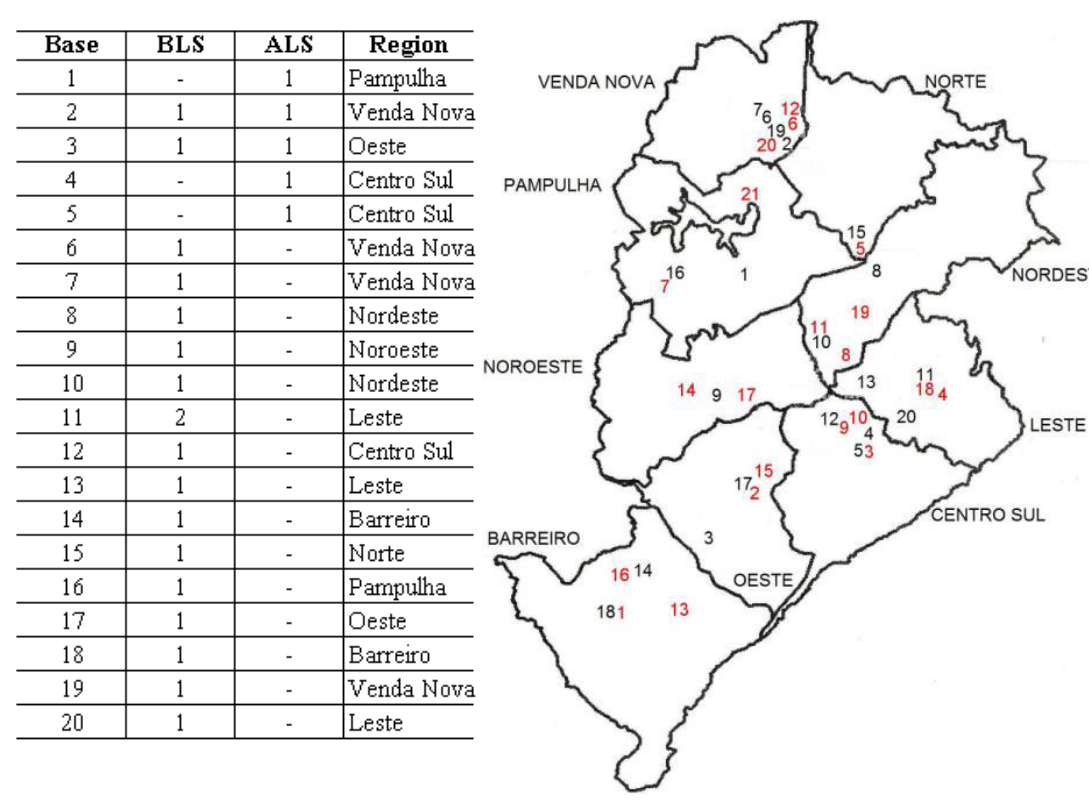

\begin{tabular}{|c|c|c|c|}
\hline $\begin{array}{l}\text { Health } \\
\text { Center }\end{array}$ & Type & Type of Incident & Region \\
\hline 1 & UPA & Clinic - Medium & Barreiro \\
\hline 2 & UPA & Clinic - Medium & Oeste \\
\hline 3 & UPA & Clinic - Medium & Centro Sul \\
\hline 4 & UPA & Clinic - Medium & Leste \\
\hline 5 & UPA & Clinic - Medium & Norte \\
\hline 6 & UPA & Clinic - Medium & Venda Nova \\
\hline 7 & UPA & Clinic - Medium & Pampulha \\
\hline 8 & UPA & Clinic - Medium & Nordeste \\
\hline 9 & Hospital & Clinic - High & Centro Sul \\
\hline 10 & Hospital & $\begin{array}{l}\text { Clinic High and } \\
\text { Trauma }\end{array}$ & Centro Sul \\
\hline 11 & Hospital & $\begin{array}{l}\text { Clinic High, } \\
\text { Trauma and }\end{array}$ & Nordeste \\
\hline 12 & Hospital & $\begin{array}{l}\text { Clinic High and } \\
\text { Trauma }\end{array}$ & Venda Nova \\
\hline 13 & Hospital & Clinic - High & Barreiro \\
\hline 14 & Hospital & Clinic - High & Noroeste \\
\hline 15 & CERSAM & Psychiatric & Oeste \\
\hline 16 & CERSAM & Psychiatric & Barreiro \\
\hline 17 & CERSAM & Psychiatric & Noroeste \\
\hline 18 & CERSAM & Psychiatric & Leste \\
\hline 19 & CERSAM & Psychiatric & Nordeste \\
\hline 20 & CERSAM & Psychiatric & Venda Nova \\
\hline 21 & CERSAM & Psychiatric & Pampulha \\
\hline
\end{tabular}

Figure 1: Spatial distribution of the bases and health centers of Belo Horizonte

Calls are received at the main base (base 5); nowadays, there are 14 workers in this base (6 phone operators, 5 doctors and 3 dispatchers). The system works with 8 Emergency Care Units (UPA), 6 hospitals and 8 Mental Health Care Centers (CERSAM). The location of these centers and hospitals are shown in Figure 1.

\subsection{Parameters of the model}

The analysis of historical data used a database containing 1,734,416 calls which were made between October 2006 and August 2009. The duration of each activity of the system was estimated from data collected directly from the Central. The samples were collected on particular days of the week and at particular hours throughout the day. Due to the lack of data, two activities (material replacement and patient hospitalization) had a service time estimate based on the perception of the officials involved and the few time samples collected.

The average speeds were $25 \mathrm{Km} / \mathrm{h}$ between 7:00am - 9:00pm, and $50 \mathrm{~km} / \mathrm{h}$ between 9:00pm 7:00am. The coefficient of the correction of Euclidean distance in Belo Horizonte was estimated at 1.366. For verification of the developed model, we used the technique of modular implementation, where each part of the model is implemented and then run separately. For each module, the response variables were analyzed in order to test their consistency with the model behavior. Users of the system also forced the occurrence of unlikely events and occurrences that cause unusual decisions in the dispatch of ambulances, such as the arrival of a call that requires an advanced unit, but whose available unit closest to the incident is basic.

The validation process, from the initial design stages of the project until its completion, was conducted with the direct involvement of system managers. The conceptual modeling of the whole system 


\section{Silva and Pinto}

and all the simplifications adopted for its computational implementation were discussed previously with the managers. The definition of variables that would be used to analyze the system performance and determine which scenarios would be evaluated were also discussed directly with the doctor in charge of the system's operation. Here, the model was implemented in Arena (Rockwell Software).

Data concerning the system's performance, such as the elapsed time between the arrival of the call and the dispatch of ambulance, travel times and the service time at the incident site, were collected directly in the system because such data was not available in the database. The developers of the system collected about 100 pieces of data for each of these measures on particular days and hours of the week. The results are shown in Tables 1 and 2.

Table 1: Waiting time and total time validation

\begin{tabular}{l|c|c|c|c}
\hline & \multicolumn{2}{|c|}{ Waiting Time [min] } & \multicolumn{2}{c}{ Total Time [min] } \\
\hline & Simulated & Real & Simulated & Real \\
\hline Minimum & 0.50 & 0.33 & 8.37 & 9.14 \\
\hline Average & 6.07 & 6.13 & 35.35 & 31.82 \\
\hline Maximum & 52.66 & 48.91 & 96.07 & 79.75 \\
\hline
\end{tabular}

Table 2: Number of dispatched units

\begin{tabular}{c|c|c|c}
\hline Month & Real & Simulated & Diference \\
\hline $\mathrm{dec} / 08$ & 7,437 & 7,453 & $-0.21 \%$ \\
\hline $\mathrm{jan} / 09$ & 7,266 & 7,470 & $-2.81 \%$ \\
\hline $\mathrm{feb} / 09$ & 6,752 & 6,722 & $0.44 \%$ \\
\hline $\mathrm{mar} / 09$ & 7,503 & 7,458 & $0.60 \%$ \\
\hline $\mathrm{apr} / 09$ & 7,281 & 7,196 & $1.17 \%$ \\
\hline may $/ 09$ & 7,528 & 7,416 & $1.49 \%$ \\
\hline jun/09 & 6,934 & 7,140 & $-2.97 \%$ \\
\hline jul/09 & 7,417 & 7,508 & $-1.23 \%$ \\
\hline aug $/ 09$ & 7,429 & 7,388 & $0.55 \%$ \\
\hline
\end{tabular}

\subsection{Scenarios Analysis and Results}

The first scenario evaluates the performance of the system when the demand for services is increased (Figure 2). Here, the demand is directly related to several factors: population size, knowledge and people's access to emergency medical services, the scope of the service, accident reduction policies, etc. The average response time also increases slightly to a $20 \%$ increase in demand for services. From this point, it will begin to grow in a more pronounced manner. If demand increases by $30 \%$, the average response time will increase by more than 17 minutes. However, if demand increases by $40 \%$, the increase in the response time will be more than twice the current value. The average waiting time to answer calls is more than twice the current value, with a $20 \%$ increase in demand. However for a $40 \%$ increase, this time has an average value of almost 30 minutes, which is totally incompatible with the desirable performance for this type of system. The size of the queue refers to the amount of calls that are waiting for the dispatch of an ambulance. This variable can be a good indicator of the relationship between the performance of operations within the Central and external operations. The scenario of a $30 \%$ increase in demand, indicates an average queue size of 4.09 and represents a congested system that does not work satisfactorily. The maximum size of the waiting queue was used to improve the understanding of the behavior of the system; the system may have an acceptable middle value for the size of the waiting queue, but has a value at peak times that is very large and unacceptable for a satisfactory operation of the system.

Another scenario was used to analyze the number of ambulances needed at each base so that the response time was less than 18 minutes for the basic units and less than 15 minutes for the advanced units. This analysis requires the use of optimization for simulation. The optimization model was therefore implemented using OptQuest for Arena (OptTek Systems). The solution was 20 basic units and 8 advanced units (with a current value of 17 basic units and 5 advanced units); here the response time was 17.97 and 14.77 minutes respectively. 


\section{Silva and Pinto}
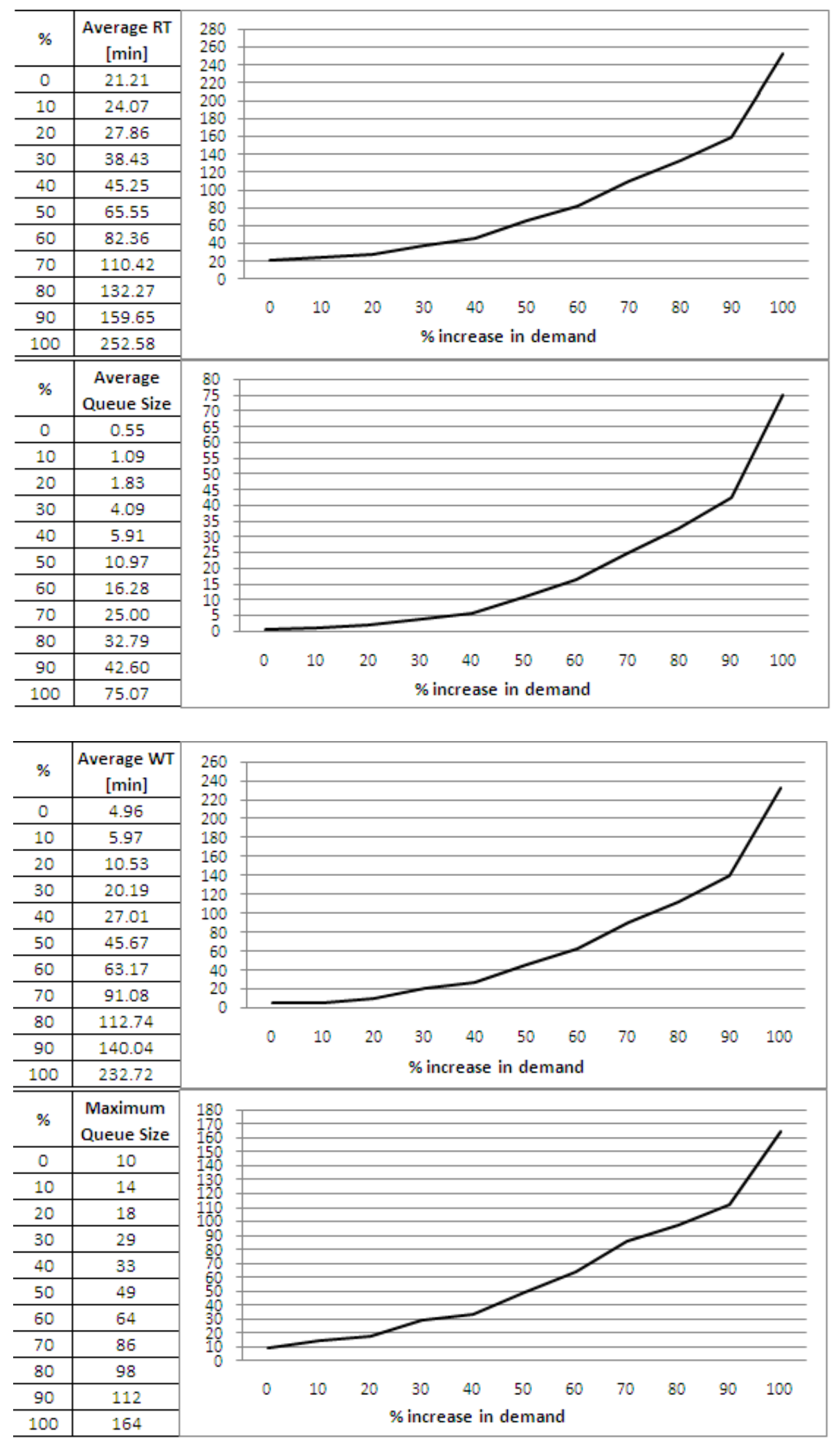

Figure 2: Impact of demand growth in the system's performance 


\section{Silva and Pinto}

\section{CONCLUSION}

The emergency medical services have a high randomness associated with the duration of the tasks that are part of the system's routine. In addition, many tasks do not have clear rules and depend on the people who are performing the task. However, the discrete event simulation is a useful tool for an analysis of these systems, and it is possible to develop models that are able to represent satisfactorily all the phenomena that are part of all the activities of the system. These models make possible the analysis of future scenarios with a statistically acceptable reliability, as was achieved with the model developed for the city of Belo Horizonte, assessing scenarios of increased demand and others to reduce the response time. Therefore, the combined use of optimization techniques and simulation improves the search for optimal settings for the system, allowing the simulation and analysis of a large number of alternatives.

\section{ACKNOWLEDGMENTS}

The authors thanks FAPEMIG (Research Support Foundation of State of Minas Gerais) for financially supporting their participation in the conferences.

\section{REFERENCES}

Aringhieri, R., G. Carello, and D. Morale. 2007. Ambulance location through optimization and simulation: the case of Milano urban area. XXXVIII Annual Conference of the Italian Operations Research Society Optimization and Decision Sciences:1-29.

Azadivar, F. 1992. A tutorial on simulation optimization. In Proceedings of the 1992 Winter Simulation Conference, ed. J. J. Swain, D. Goldsman, R. C. Crain, and J. R. Wilson, 198-204. Piscataway, New Jersey: Institute of Electrical and Electronics Engineers, Inc.

Ball, M. O. and L.F. Lin. 1993. A reliability model applied to emergency service vehicle location. Operations Research 41:18-36.

Brailsford, S. C. 2007. Tutorial: advances and challenges in healthcare simulation modeling. In Proceedings of the 2007 Winter Simulation Conference, ed. S. G. Henderson, B. Biller, M.-H. Hsieh, J. Shortle, J. D. Tew, and R. R. Barton, 1436-1448. Piscataway, New Jersey: Institute of Electrical and Electronics Engineers, Inc.

Campbell, J. P., M. C. Gratton, J. A. Salomone, and W. A. Watson. 1993. Ambulance arrival to patient contact: the hidden component of prehospital response time intervals. Annals of Emergency Medicine 22:1254-1257.

Church, R. L., and C. Revelle. 1974. The maximal covering location problem. Papers of the Regional Science Association 32:101-118.

Cummins, R. O. 1989. From concept to standard-of-care? Review of clinical experience with automated external defibrillators. Annals of Emergency Medicine. 18:1269-1275.

Cummins, R. O., M. S. Eisenberg, A. P. Hallstrom, and P. E. Litwin. 1985. Survival of out-of-hospital cardiac arrest with early initiation of cardiopulmonary resuscitation. The American Journal of Emergency Medicine 3:114-119.

Eldabi, T., and Y. Young. 2007. Towards a framework for healthcare simulation. In Proceedings of the 2007 Winter Simulation Conference, ed. S. G. Henderson, B. Biller, M.-H. Hsieh, J. Shortle, J. D. Tew, and R. R. Barton, 1454-1460. Piscataway, New Jersey: Institute of Electrical and Electronics Engineers, Inc.

Fetter, R. B., and J. D. Thompson. 1966. Patients waiting time and doctors idle time in the outpatient setting. Health Services Research 1:66-90.

Fitzsimmons, J. A. 1971. An emergency medical system simulation model. In Proceedings of the 5th Conference on Winter Simulation, 18-25. Piscataway, New Jersey: Institute of Electrical and Electronics Engineers, Inc. 
FU, M. C. 2002. Simulation for optimization: theory vs. practice. INFORMS Journal on Computing 14(3):192-215.

Koch, O., and H. Weigl. 2003. Modeling ambulance service of the Austrian redcross. In Proceedings of the 2003 Winter Simulation Conference, ed. S. Chick, P. J. Sánchez, D. Ferrin, and D. J. Morrice, 1701-1706. Piscataway, New Jersey: Institute of Electrical and Electronics Engineers, Inc.

Maxwell, M. S., S.G. Henderson, and H. Topaloglu. 2009. Ambulance redeployment: an approximate dynamic programming approach. In Proceedings of the 2009 Winter Simulation Conference, ed. M. D. Rossetti, R. R. Hill, B. Johansson, A. Dunkin and R. G. Ingalls, 1850-1860. Piscataway, New Jersey: Institute of Electrical and Electronics Engineers, Inc.

Novaes, A. G. Sistemas logísticos: transporte, armazenagem e distribuição física de produtos. São Paulo: Editora Edgard Blucher, 1989. 372 p.

Oliveira, L. K., and M.B. Gonçalves. 2004. Metodologia para avaliar centro de emergência: aplicação ao centro de emergência da Polícia Militar de Santa Catarina. Revista Produção Online 4(2).

Shih, C. L. and S. Su. 2003. Modeling an emergency medical services system using computer simulation. International Journal of Medical Informatics 72(3):57-72.

Singer, M. and P. Donoso. 2008. Assessing an ambulance service with queuing theory. Computers and Operations Research 35:2549-2560.

Takeda, R. A., J.A. Widmer, and R. Morabito. 2001. Uma proposta alternativa para avaliação do desempenho de sistemas de transporte emergencial de saúde brasileiros. Transportes 9(2):9-27.

Timerman, S., M.M.C. Gonzalez, A.C. Zaroni, and j.A.F. Ramires. 2006. Emergency medical services: Brazil. Resuscitation 70:356-359.

Toregas, C., R. Swain, C. Revelle, and L. Bergman. 1971. The location of emergency service facilities. Operations Research 19:1363-1373.

\section{AUTHOR BIOGRAPHIES}

LUIZ RICARDO PINTO has a PhD in Production Engineering /Operational Research (COPPE/UFRJ 1999), an MSc. in Engineering (UFOP - 1988) and a bachelors in Mining Engineering (UFOP - 1983).

He has worked as consultant in several projects concerning operations research. He is an Associated Professor of undergraduate and graduate courses of Industrial Engineering at the Federal University of Minas Gerais - UFMG and can be contacted by email at <lui z@dep. ufmg. br $>$.

PEDRO MARINHO SIZENANDO SILVA has an MSc. in Production Engineering (UFMG - 2010) and a bachelors in Production Engineering (UFMG - 2007). He has worked with simulation models applied to health systems, industrial processes and the flow of passengers in airport terminals and can be contacted by email at <pedromarinhosilvalgmail.com>. 